\title{
P09-2 I LB. Deep sequencing of HIV-I from acute infection: low initial diversity, and rapid but variable CTL escape
} W Fischer*1, B Keele ${ }^{2}$, T Bhattacharya ${ }^{3}$, C Lo ${ }^{1}$, E Giorgi ${ }^{1}$, P Hraber ${ }^{1}$, T Leitner ${ }^{1}$, C Han ${ }^{4}$, C Gleasner ${ }^{4}$, L Green ${ }^{4}$, B Hahn ${ }^{5}$, G Shaw ${ }^{6}$, B Haynes ${ }^{7}$ and B Korber ${ }^{1}$

\begin{abstract}
Address: ${ }^{1}$ Los Alamos National Laboratory, Los Alamos, NM, USA, ${ }^{2}$ Dept. of Medicine, Univ. of Alabama at Birmingham, Birmingham, AL, USA, ${ }^{3}$ Group T-2, Los Alamos National Laboratory, Los Alamos, NM, USA, ${ }^{4}$ Group B-6, Los Alamos National Laboratory, Los Alamos, NM, USA, ${ }^{5}$ Depts. of Medicine and Microbiology, Univ. of Alabama at Birmingham, Birmingham, AL, USA, ${ }^{6}$ Division of Hematology/Oncology, Univ. of Alabama at Birmingham, Birmingham, AL, USA and ${ }^{7}$ Duke Human Vaccine Institute, Duke University Medical Center, Durham, NC, USA

* Corresponding author
\end{abstract}

from AIDS Vaccine 2009

Paris, France. 19-22 October 2009

Published: 22 October 2009

Retrovirology 2009, 6(Suppl 3):P40I doi:I0.I I86/I742-4690-6-S3-P40 I

This abstract is available from: http://www.retrovirology.com/content/6/S3/P40 I

(c) 2009 Fischer et al; licensee BioMed Central Ltd.

\section{Background}

Events in early HIV infection are crucial to understanding several questions vital to vaccine development -- How many viral variants establish initial infection? How soon does a T-cell response to the virus appear? How quickly does the virus evolve to escape immune responses, and what escape routes does it use? Deep sequencing allows characterization of viral populations in unprecedented detail, illuminating these questions.

\section{Methods}

We sequenced acute-infection samples from three patients previously characterized by conventional sequencing, targeting specific regions known to have undergone rapid immune escape. Using multiplex 454 sequencing of PCR products amplified with tagged primers, we obtained sequence from three timepoints per patient (from escape regions and the V3 loop). We employed novel methods to reduce 454 sequence errors, and used statistical modeling to evaluate the likelihood of single-virion versus multipleviron infection.

\section{Results}

454 sequencing yielded thousands of sequences from timepoints before, during, and after immune escape. Protein entropy confirmed epitope locations; the frequency of variant peptides revealed patterns of viral evolution. In one patient (SUMA), variation developed explosively (escape variants comprising $75 \%$ of the timepoint-three virus had only $0.05 \%$ combined frequency at timepoint two); in a second ( $\mathrm{CH} 40)$, rapid divergence generated extensive variation, but a dominant form's emergence greatly reduced population variability (a selective sweep); in the third patient (WEAU), variation within the epitope developed less rapidly, and no single escape mutant had become dominant by the last timepoint.

\section{Conclusion}

Far greater diversity was observed than with conventional sequencing, but evolutionary modeling of variant distribution supported the hypothesis that a single virus established infection. Escape mutants that subsequently became dominant were detected while very rare, and could become established in as little as three weeks. In escaping from CTL pressure, HIV-1 explored a very large number of mutants, but the rate of population turnover necessarily depends upon net selective pressure. 